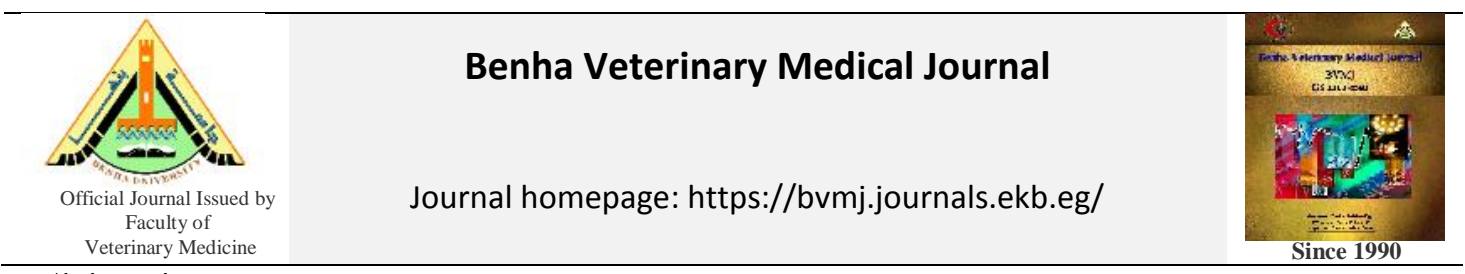

Mini-Review

\title{
Natural antifungal agents for food preservation
}

Ekbal A Ibraheem . Hend A Elbarbary and Mahmoud M Hammad

Food Quality Control Program (FQCP), Faculty of Veterinary Medicine, Benha University.

\section{ARTICLE INFO}

\section{Keywords}

Natural antifungal agents

food preservation

Food spoilage

Received 21/03/2020

Accepted 04/09/2020

Available On-Line

$01 / 10 / 2020$

\begin{abstract}
Food spoilage is a major issue for the food industry, leading to food waste, substantial economic losses for manufacturers and consumers, and a negative impact on brand names. Among causes of food spoilage, fungal contamination can be encountered at various stages of the food chain (e.g., post-harvest, during processing or storage). Fungal development leads to food sensory defects varying from visual deterioration to noticeable odor, flavor, or texture changes but can also have negative health impacts via mycotoxin production by some molds. In order to avoid microbial spoilage, and thus extend product shelf life, different treatments, including fungicides and chemical preservatives, are used. In parallel, public authorities encourage the food industry to limit the use of these chemical compounds and develop natural methods for food preservation.
\end{abstract}

\section{PUBLIC HEALTH HAZARD}

Growth of commonly occurring filamentous fungi in foods may result in production of toxins known as mycotoxins, which can cause a variety of ill effects in humans, from allergic responses to immunosuppression and cancer. The most important mycotoxins are aflatoxins, ochratoxin A, fumonisins, trichothecenes and zearalenone.

Aflatoxins are potent carcinogens and, in association with hepatitis B virus, are responsible for many thousands of human deaths per annum, mostly in non-industrialized tropical countries.

Ochratoxin A is a probable carcinogen and may cause urinary tract cancer and kidney damage in people from northern and eastern Europe.

Fumonisins appear to be the cause of esophageal cancer in southern Africa, parts of China and elsewhere. Trichothecenes are highly immunosuppressive and zearalenone causes estrogenic effects in animals and man. Current available records and statistics do not reflect the major role played by mycotoxins in mortality attributable to food-borne micro-organisms (Smith J et al 1985). The symptoms of mycotoxicosis are almost as diverse as the chemical structures of the compounds themselves. Some compounds may elicit few symptoms until death results, while others may produce severe effects including skin necrosis, leucopoenia and immunosuppression. Doses producing chronic disease are usually far below those responsible for acute effects, and so long-term effects such as cancer or tumor induction are undetected at the time of ingestion and, indeed, may remain so until disease is quite advanced (Joffe et al., 1979). Many of the toxigenic fungi are ubiquitous and, in some cases, apparently have a strong ecological link with human food supplies. The natural fungal flora existing in conjunction with food production is dominated by three genera: Aspergillus, Fusarium and
Penicillium. Fusarium species are destructive pathogens on cereal crops and other commodities, and produce mycotoxins before, or immediately after, harvest. Certain species of Aspergillus and Penicillium are also plant pathogens or commensals, but these genera are more commonly associated with commodities and foods during drying and storage

\section{NATURAL ANTIFUNGAL AGENTS IN FOOD PRESERVATION}

\section{Ethanolic plant extracts}

In an investigation by Shetty et al. (1993), out of the 22 ethanolic extracts, clove extract was found to be highly active against Scopulariopsis sp., Aspergillus luchuensis, A. flavus, Penicillium oxalicum, Rhizopus stolonifer and Mucor sp. Several workers have earlier reported that clove ethanolic extract showed antimycotic activity against fungal genera such as Aspergillus, Penicillium, Rhizopus, Cladosporium and Saccharomyces. This activity may be due to the presence of eugenol and caryophyllene (Shetty et al., 1993).

\section{Garlic ethanolic extract}

Garlic ethanolic extract inhibited the growth of 10 fungal isolates except Penicillium oxalicum isolate. The inhibitory activity of Allium vegetable extracts against molds have been reported by numerous authors. Allicin, thiosulphonate and other compounds have been shown to have fungistatic activity against Aspergillus spp. such as A. flavus, A. fumigatus, A. terreus and Penicillium chrysogenum. Several studies have reported that garlic extract can inhibit the growth of bacteria, fungi, viruses in culture media and food systems and it has also been shown to possess insecticidal, antiparasitic and antitumor properties. Several ajoene compounds which are derivatives of allicin,

\footnotetext{
* Corresponding author: Mahmoud M Hammad, Graduate student, Food Quality Control Program (FQCP), Faculty of Veterinary Medicine, Benha University.
} 
obtained from garlic with ethanol extraction have been found to be very inhibitory against $\mathrm{A}$. niger and Candida albicans. Other compounds from garlic such as garlicin and thiosulfonate have also been reported to possess antimicrobial properties (Khalil et al., 2001)

\section{Ginger ethanolic extract}

Ginger ethanolic extract inhibited the growth of all the test fungal isolates with zone of mycelial growth inhibition of $30 \%$ to $100 \%$. It may be due the presence of caprylic acid, zingerone, shogaols and gingerols. Turmeric ethanolic extract showed inhibitory activity against only two molds namely R. stolonifer (25\% to $40 \%$ ) and Mucor sp. (40\%). Turmeric has also been shown to induce predominant antifungal activity due to the presence of turmeric oil and curcumin. However, there are reports of occurrence of antifungal activity against other molds such as Aspergillus niger and Penicillium digitatum and a niger, A. flavus, $\mathrm{P}$. javanium, Curvularia oryzae and Trichophyton mentagrophytes (Arora et al., 1999)

\section{Cinnamon ethanolic extract}

Cinnamon ethanolic extract was found to be active against A. luchuensis only. Out of the 11 food-associated fungi tested, the activity of cinnamon extract was found against Aspergillus flavus and A. parasiticus. The inhibitory activity of cinnamon may be due to the presence of cinnamaldehyde and eugenol. The fungistatic or fungicidal effect of spices is due to the inhibitory action of natural products and the mechanisms involved are cytoplasm granulation, cytoplasmic membrane rupture and inactivation and/or inhibition of intracellular and extracellular enzymes (Shetty et al., 1993).

These biological events could take place separately or concomitantly culminating with mycelium germination inhibition. It was also reported that plant lytic enzyme act on the fungal cell wall causing breakage of $B-1,3$ glycan, $B-$ 1,6 , glycan and chitin polymer

\section{Cumin ethanolic extract}

Cumin ethanolic extract showed inhibitory activity against Penicillium oxalicum isolate. This activity may be due to the presence of cumin volatile oil and cumin aldehyde compound (Shetty et al., 1993).

\section{Clove ethanolic extract}

Out of the 22 extracts evaluated, clove ethanolic extract has been found to be the best antifungal agent showing $70 \%$ to $100 \%$ mycelial growth inhibition of different molds tested as compared to acetic acid, commonly used preservative tested as positive control where the mycelial growth inhibition ranged between $60 \%$ and $100 \%$ (Phyllis and James, 2000).

\section{ANTIFUNGAL MICROORGANISMS}

\section{Lactic acid bacteria}

Lactic acid bacteria (LAB) encompasses a large and heterogeneous group of Gram-positive, low-GC, acidtolerant bacteria, which produce lactic acid as the major metabolic end product of carbohydrate fermentation. LAB belongs to the Lactobacillus lesorder, which include 6 families, 36 genera, and more than 200 species. They are found in various biotopes such as environment, plants, human, and animal microbiota. (Cray et al., 2013). They are largely used in the manufacture of a variety of fermented foods, where they contribute to improve shelflife, organoleptic properties, and nutritional value.

\section{Propionic bacteria}

Dairy propionic bacteria (PAB) are also considered as antifungal candidates. The Propionibacterium genus, belonging to the Actinobacteria class, is a Gram-positive, high-GC content bacteria divided into 'cutaneous' and 'classical' (also referred to as 'dairy') propionibacteria based on their main isolation biotopes (Stackebrandt et al., 2016). PAB and related species taxonomy has recently been reconsidered, with in particular 'dairy' PAB separated in the Propionibacterium genus consisting of $P$. freudenreichii, the main species used in cheese-making, and three other species, and a novel genusAcidipropionibacterium-which encompasses the former species P. acidipropionici, P. thoenii, P. jensenii, and three other species . In a large in vitro screening study on 197 dairy PAB strains, 13 strains-including 9 P. jensenii, 2P. acidipropionici, and 2 P.thoenii-showed high antifungal activity against various yeast and mold species. (Scholz et al .2016).

\section{Bacillus and other bacteria}

Some recent bio-protection assays performed in situ using Bacillus species cells, endospores, CFS, or purified peptides on postharvest fruits illustrate the potential activities that they can exhibit . Bacillus sp. strains present a large activity spectrum against pathogenic fruit molds from the Aspergillus, Alternaria, Beltraniella, Botryosphaeria, Botrytis, Monilinia, Colletotrichum, Fusarium, Fusicoccum, Penicillium, Phomopsis, Phoma, Rhizoctonia, and Rhizopus genera. In another study, whole cultures of two B. amyloliquefaciens strains sprayed on Chinese jujube fruit inoculated with spores of three key pathogens of this fruit, Phoma destructiva, Alternaria alternata and Fusicoccum spp., reduced the disease incidence and induced different kinds of hyphal alterations on the fungal targets .

\section{Essential oils}

In the food industry there is great interest in essential oils (Eos) derived from MAPs due to their property of controlling the growth of pathogenic microorganisms, such as Fusarium spp., Aspergillus spp., among others, which have been reported as causative agents of diseases caused by food and/or decomposition of the same. The EOs have been used against a broad range of fungal pathogens. Clove essential oil showed a MIC value of 0.062 and 0.125 $\%$ (v/v) against Candida albicans , respectively. Rosemary EO is also effective against C. albicans and A. niger, but with MIC values of 0.25 and $1.0 \%$ (v/v), respectively (Swamy et al., 2016). The EOs of thyme and clove completely inhibited the mycelium growth of Aspergillus flavus. The major chemical compound of clove, eugenol, was proved to produce permanent damage to the cells of $\mathrm{C}$. albicans and was considered an efficient antifungal agent, with a MIC value of $1.0 \% \mathrm{v} / \mathrm{v}$. EOs of clove, cinnamon and oregano were effective against Aspergillus parasiticus and Fusarium moniliforme, because they caused damage in mycelium growth and mycotoxin-producing ability (Ebani et al., 2016). Essential oil from Ageratum conyzoides successfully controlled rotting of mandarins by blue mold and increased mandarin shelf-life by up to 30 days (Dixit $e t$ al., 1995 


\section{NATURAL ANTIFUNGAL PEPTIDES/ PROTEINS}

\section{Thaumatin-like proteins}

TLPs are 21 to $26 \mathrm{kDa}$ proteins, with 16 conserved cysteine residues forming eight disulfide linkages. Their structure is made of three folded domains, with an interesting basic cleft, probably involved in the antifungal action. TLPs range of activity includes yeasts and filamentous fungi, such as Fusarium spp. and S. cerevisiae. Osmotin and zeamatin, expressed in tobacco and maize, respectively, are among the most well described TLPs (Garcia-Casado et al., 2000). They provide protection toward abiotic stresses and invading phytopathogens. Osmotin inhibits the growth of S. cerevisiae (IC50 $=0.3 \mu \mathrm{M}$; (Yun et al., 1998), Fusarium solani, and Fusarium oxysporum (IC50 $=67 \mu \mathrm{g} / \mathrm{mL} \quad$ (De Freitas et al., 2011) by using different mechanisms depending on the fungal targets. TLPs' main mechanism is believed to be due to their ability to create trans-membrane pores. TLPs demonstrate variable activities against fungal species, which has been attributed to specific composition of fungal cell wall and membrane. In addition to the low concentration required for their activity, the structure of TL proteins imparts a great stability to heat- and proteolytic treatment of under varied $\mathrm{pH}$ (Roberts and Selitrennikoff, 1990).

\section{Thionins}

Thionins consist of two antiparallel $\alpha$-helices and one antiparallel double stranded $\beta$-sheet connected by three or four disulfide bridges, forming a characteristic stable gamma fold. Thionins are hydrophobic and display their antifungal activity via interactions of the hydrophobic and/or polar residues with membrane elements, possibly leading to lysis of the fungal cell membrane (De Lucca et al., 2005). Capsicum annum thionin-like peptide (CaThi) is an inhibitor of several Candida species and S. cerevisiae (Taveira et al., 2014, 2016). Yeasts treated with CaThi show structural damage to their membrane and eventual permeabilization. In S. cerevisiae, CaThi interacts with components of the plasma membrane, which alters the proton gradient and inhibits acidification of the medium, ultimately inducing an increase in reactive oxygen species (ROS) formation. CaThi has been suggested to have cytoplasmic targets in C. tropicalis cells (Taveira et al., 2016). Although most antifungal thionins, such as viscotoxins (Giudici and Antonio Poveda, 2006), show toxicity to mammalian cells, some thionins and pseudothionins are considered safe (Srikanth and Chen, 2016). Thestability of thionins is ensured by the SS bonds and the residue arginine in position 10 , as it is critically involved in hydrogen bonding (HB) with associated secondary structures. However, thionins' ability to permeabilize fungal membrane is inhibited by the presence of salts, a property that might limit their utility as food agents.(Rao et al., 1994).

\section{Snakins}

Snakins were originally isolated from potato, but have recently been found in other species, such as tomato (Solanum lycopersicum). These $7 \mathrm{kDa}$-peptides contain a stable two $\alpha$-helical structure stabilized by six disulfide bonds, with an essential C-terminus. This conserved Cterminus ensures biological activity and stability of the

peptide. Upon tissue degradation or invasion, potato (Solanum tuberosum cv Jaerla) tubers express a peptide termed snakin-2 (StSN2). StSN2 shows activity toward several phytopathogens such as Fusarium spp. and A. niger (IC50 $=1$ to $20 \mu \mathrm{m})$. Other snakins, such as a 64-residue homolog of StSN2 identified in French bean or snakin Z isolated from Jujuba fruits, are active against A. niger (Daneshmand et al., 2013). Another 66-residue snakin-2 (Sn2) found in tomato shows a broad-spectrum activity, including against $\mathrm{S}$. cerevisiae $(\mathrm{MIC}=4.25 \mu \mathrm{M})$. The mechanism underlying the bioactivity of $\mathrm{Sn} 2$ includes its agglomeration into single yeast cells prior to killing and the formation of pores in the membrane of target cells. However, snakins are not predicted to act via membrane permeabilization, since they did not exhibit membrane disruptive effect on liposomes. The major hurdles for food application is the alteration of their activity in the presence of cations, as shown with $\mathrm{Sn} 2$ against $\mathrm{S}$.cerevisiae. Moreover, Sn2 shows $20 \%$ hemolyticactivityat $17 \mu \mathrm{M}$ and $100 \%$ at $35 \mu \mathrm{M}$ (Herbel et al., 2015)

\section{Albumins}

$2 \mathrm{~S}$ Albumins are storage proteins used by seeds as a source of nitrogen during germination. 2S Albumins play a protective role in plants by exhibiting antifungal activity, as shown for $2 \mathrm{~S}$ albumins isolated from Malva parviflora and radish (Raphanus sativus) seeds.. 2S Albumins are synthesized as a polypeptide of 18 to $21 \mathrm{kDa}$ in response to pathogens and cleaved into two peptides as a posttranslational modification (PTM). These two peptides represent the small and large chain and present similar characteristics to other AFPs, such as richness in cationic residues and presence of disulfide bonds. 2S Albumins permeabilize the fungal membrane, leading to an impairment of the H+/ATPase and inhibition of the glucose stimulated acidification of the media. This mechanism was identified for the $2 \mathrm{~S}$ albumin, $\mathrm{Ca}-\mathrm{Alb}$, isolated from Capsicum annuum (Ribeiro et al., 2012). Cells treated with the $2 \mathrm{~S}$ albumin Pf-Alb exhibit an apoptotic-like behavior, including shrinking of the cells so land cell wall deformation. In addition, the growth of yeasts K. marxianus and C. parapsilosis is inhibited via NO induction in presence of Ca-Alb. Unfortunately, allergens, which limits their use in food preservation. The presence of eight Cys residues and several $\alpha$-helices ensures a high stability of the protein structure (Ullah et al., 2015)

\section{Knottins}

Plant knottins are characterized by ahigh stability to heat, chemical, or enzymatic treatment. Cyclotides are a subfamily of the knottins and known for their high stability. These circular peptides of 28 to 37 residues have been identified in bacteria, plants, and animals. A head-to-tail cyclical backbone and three intramolecular disulfide bonds confer them a supplementary stability. Cyclotides are classified into three subfamilies: Moebius cyclotides, which contain a $180^{\circ}$ backbone twist, the bracelets cyclotides and hybrid Moebius cyclotides, also named kalata first (Heitz et al., 2008). Isolated from Oldenlandia affinis Coffee plants contain four cyclotides (Kalata, circulin and cyclopsychotride) with activity against Candida kefyr (MIC14.0 to $29.0 \mu \mathrm{M}$ ) and C. tropicalis (MIC 21.4 to 56.5 
$\mu \mathrm{M})$. Membrane interactions between cyclotides and phospholipids, and a cell penetrating behavior, may be involved in the various biological activities, but their mechanism of action remains unknown.

\section{CONCULSION}

The increasing societal demand for less processed and more natural food products-while conserving those products' quality, safety, and shelf-life, has raised the question of chemical preservative replacement. In this context, bacteria and fungi as well as their metabolites are natural alternatives of interest for use in food as bioprotective tools to fight fungal spoilage and to answer consumer demands and legislation. From an applied point of view, the difference between the number of studies and the number of available microbial cultures indicates that efforts are needed to facilitate their application in food commodities. One of the main aspects concerns the crucial role of in situ studies using adapted fungal targets during antifungal activity screening or confirmation processes. Also, safety assessment, organoleptic neutrality, and activity stability of the bioprotective cultures need to be evaluated prior to marketing. From a cognitive point of view, while antifungal compounds have been widely studied, and have generally been shown to act synergistically, there is still a lack of knowledge concerning the overall picture as to what molecules are involved and their action mechanism(s). The combination of pertinent biochemical analytic tools and omics methods should enable us to decipher antifungal action mechanisms, potentially identifying new levers for antifungal activity. Finally, if finding natural antifungal agents is a key factor, it can only be considered in good practices and within the HACCP context as one of the hurdle technologies to prevent fungal spoilage

Intensive research on new ways to fight human and plant fungal pathogens has highlighted the potential of AFPs in the immune system of organisms, including plants. This review, and numerous reports, highlights the promising activity of these plant AFPs against food-related fungi EOs are natural substances extracted from medicinal and aromatic plants, commonly by distillation processes. These compounds have an important role in food preservation contributing to safety and shelf-life extension of food products. The improvement in food safety is due to the inhibition of pathogenic microbial growth and reduction of biogenic amines, mainly in meat and meat and dairy products, as a consequence of the inhibited growth of spoilage microorganisms. The extension of food products' shelf-life results from enzymatic reduction, mainly due to their antioxidant activity

Bacteriocins has proved potential in preventing fungal food spoilage. 1,00,000 AU/g of Micro-Cel of bacteriocin

Natamycin has been approved as a food additive in many countries, but in most the approval is for use in the dairy industry only. In a number of countries, a wider use is permitted, for example, in the meat industry or as a general food additive.

\section{REFERENCES}

1. Arora, D. S. (1999): Some Indian spices and their antimicrobial properties. In: From Ethnomycology to Fungal Biotechnology: Exploiting Fungi from Natural Resources for
Novel Products. Singh, J. and Aneja, K.R. (Eds.). Kluwer Academic/Plenum Publishers, New York.

2. Cray, J.A.; Bhaganna, P.; Singhal, R.S.; Patil, S.V.; Saha, D.; Chakraborty, R; Timson, D.J.; Hallsworth, J.E 2013. Modern fungicides and antifungal compounds VII. In Proceedings of the 17th International Reinhardsbrunn Symposium, Friedrichroda, Germany; DPG Spectrum Phytomedizin; DPG-Verl: Braunschweig, Germany

3. De Lucca, A. J., Cleveland, T. E., \& Wedge, D. E. (2005). Plant-derived antifungal proteins and peptides. Canadian Journal of Microbiology 51(12):1001-14.

4. De Freitas, C. D. T., Lopes, J. L. D. S., Beltramini, L. M., De Oliveira, R. S. B., Oliveira, J. T. A., \& Ramos, M. V. (2011). Osmotin from Calotropis procera latex: New insights into structure and antifungal properties. Biochimica et Biophysica Acta - Biomembranes, 1808(10): 2501-07.

5. Daneshmand, F., Zare-Zardini, H., \& Ebrahimi, L. (2013). Investigation of the antimicrobial activities of Snakin-Z, a new cationic peptide derived from Zizyphus jujuba fruits. Natural Product Research, 27(24): 2292-96.

6. Ebani VV, Nardoni S and Bertelloni F 2016. Antibacterial and antifungal activity of essential oils against some pathogenic bacteria and yeasts shed from poultry. Flavour and Fragrance Journal, 31(4), 302-309

7. Garcia-Casado, C., Collada, C., Allona, (2000). Characterization of an apoplastic basic thaumatin-like protein from recalcitrant chestnut seeds. Physiologia Plantarum, 110(2), 172-180

8. Heitz, A., Avrutina, O., Le-Nguyen, D., Diederichsen, U., Hernandez, J.-F., Gracy, J., ... Chiche, L. (2008). Knottin cyclization: Impact on structure and dynamics. BMC Structural Biology , 12; 8:54.

9. Herbel, V., Sch“afer, H., \& Wink, M. (2015). Recombinant production of snakin-2 (an antimicrobial peptide from tomato) in E. coli and analysis of its bioactivity. Molecules, . 20(8):14889-901.

10. Joffe, A.Z. 1969. The mycoflora of fresh and stored groundnut kernels in Israel. Mycopathol. Mycol. Appl. 39, 255-264

11. Khalil ARM 2001. Phytofungitoxin properties in the aqueous extracts of some plants. Pakistan Journal of Biological Sciences, 4 (4): 392-394

12. Phyllis B. and James B 2000: Prescription for Nutritional Healing. 3rd ed., Avery Publishing Company, America

13. Ribeiro, S. F. F., Taveira, G. B., Carvalho, A. O., Dias, G. B., Cunha, M. Da, Santa-Catarina, C., Gomes, V. M. (2012). Antifungal and other biological activities of two $2 \mathrm{~S}$ albuminhomologous proteins against pathogenic fungi. Protein Journal, 31(1):59-67.

14. Roberts, W. K., \& Selitrennikoff, C. P. (1990). Zeamatin, an antifungal protein from maize with membranepermeabilizing activity. Journal of General Microbiology, 136(9) https://doi.org/10.1099/00221287-136-9-1771

15. Rao, A. G., Hassan, M., \& Hemple, C. (1994). Structurefunction validation of high lysine analogs of $\alpha$-hordothionin designed by protein modeling. Protein Engineering, Design and Selection, Volume 7, Issue 12, December 1994, Pages 1485-1493, https://doi.org/10.1093/protein/7.12.1485

16. -Shetty RS, Singhal RS and Kulkarni PR 1993. Antimicrobial properties of cumin. World Journal of Microbiology and Biotechnology, 10, 232-233

17. Stackebrandt, E.; Cummins, C.S.; Johnson, J.L.2016: Family Propionibacteriaceae: The genus Propionibacterium. In The Prokaryotes. Dworkin, M., Falkow, S., Rosenberg, E., Schleifer, K.-H., Stackebrandt, E. (Eds). Springer: New York, NY, USA

18. Swamy MK, Akhtar MS, Sinniah UR 2016. Antimicrobial Properties of Plant Essential Oils against Human Pathogens and Their Mode of Action: An Updated Review. Evidencebased complementary and alternative medicine, 3012462. doi: 10.1155/2016/3012462. Epub 2016 Dec 20.

19. Taveira, G. B., Carvalho, A. O., Rodrigues, R., Trindade, F. G., Da Cunha, M., \& Gomes, V. M. (2016). Thionin-like peptide from Capsicum annuum fruits: Mechanism of action 
and synergism with fluconazole against Candida species. BMC Microbiology 27; 16:12.

20. Taveira, G. B., Da Motta, O. V., Machado, O. L. T., Rodrigues, R., Carvalho, A. O., Teixeira-Ferreira, A., Gomes, V. M. (2014). Thionin-like peptides from Capsicum annuum fruits with high activity against human pathogenic bacteria and yeasts. Biopolymer; 102(1): 30-9.
21. Ullah, A., Mariutti, R. B., Masood, R., Caruso, I. P., Gravatim Costa, G. H., Millena De Freita, C., Arni, R. K. (2015). Crystal structure of mature $2 \mathrm{~S}$ albumin from Moringa oleifera seeds. Biochemical and Biophysical Research Communications, 468(1-2): 4-11.

22. Yun, D. J., Ibeas, J. I., Lee, H., Coca, M. A., Narasimhan, M L., Uesono, Y., ... Bressan, R. A. (1998). Osmotin, a plant antifungal protein, subverts signal transduction to enhance fungal cell susceptibility. Molecular Cell, 1(6): 807-17 